\title{
Feasibility of using microbeads with holographic barcodes to track DNA specimens in the clinical molecular laboratory
}

We demonstrate the feasibility of using glass microbeads with a holographic barcode identifier to track DNA specimens in the molecular pathology laboratory. These beads can be added to peripheral blood specimens and are carried through automated DNA extraction protocols that use magnetic glass particles. We found that an adequate number of microbeads are consistently carried over during genomic DNA extraction to allow specimen identification, that the beads do not interfere with the performance of several different molecular assays, and that the beads and genomic DNA remain stable when stored together under regular storage conditions in the molecular pathology laboratory. The beads function as an internal, easily readable specimen barcode. This approach may be useful for identifying DNA specimens and reducing errors associated with molecular laboratory testing. 
1 Jason D. Merker, Naomi O'Grady, Linda Gojenola, Mai Dao, Ross Lenta, Joanne M. Yeakley*,

2 Iris Schrijver

3

4 Department of Pathology, Stanford University School of Medicine, Stanford, CA, USA (JDM,

5 LG, MD, IS)

6 Illumina, Inc., San Diego, CA, USA (NO, RL, JMY)

7 *Current address: 255Xpress, Inc., La Jolla, CA, USA

8

9

10 Corresponding author:

11 Iris Schrijver

12 Department of Pathology, Stanford University School of Medicine

13 Stanford, CA 94304

14 Phone: 650-724-2403; Fax: 650-724-1567

15 ischrijver@stanfordmed.org 


\section{Introduction}

17 Events that negatively impact, or could negatively impact, patient care because of quality concerns with DNA-based clinical genetic testing are rare, occurring in $<0.5 \%$ of tests performed

19 (Hofgartner \& Tait 1999). Despite this low rate of error, further reduction remains a priority of 20 clinical laboratories. Errors can occur in all phases of testing (pre-analytical, analytical, and 21 post-analytical), with the majority of problems occurring in the pre-analytical phase. This 22 emphasizes the importance of quality assurance in all phases of testing. In this brief report, we 23 describe the use of small glass microbeads containing a unique numeric code to barcode DNA 24 eluates from peripheral blood specimens. The beads can be added directly to the peripheral 25 blood specimens and are carried through automated DNA extraction protocols that use magnetic 26 glass particles. A bead reader system can identify the bead number in the specimen, which can function as an internal barcode for specimen identification. This can be used to check the identity of DNA specimens that, for example, give unexpected results. Likewise, this approach could be used to check specimens at a set interval as part of the laboratory quality assessment program. Incorporation of this microbead system directly into blood draw tubes could be used to

31 immediately barcode the specimen very early in the pre-analytical phase of testing. Preliminary

32 data suggest that this approach can be used to track DNA specimens without interfering with DNA storage or downstream molecular testing. With the increased use of nucleic acid testing to 34 guide precision or personalized medicine, this method provides a unique approach to decrease laboratory error. 
39 The use of peripheral blood specimens in this study was approved by a Stanford University IRB.

$40 \quad$ VeraCode glass microbeads

41 VeraCode microbeads were provided by Illumina, Inc. (San Diego, CA) in microcentrifuge tubes,

42 each containing approximately 40,000 beads in 70\% ethanol. VeraCode microbeads are

43 cylindrical glass beads measuring 240 microns in length by 28 microns in diameter. A digital

44 holographic element containing a numeric code is embedded within the beads serving as a

45 unique identifier. When excited by a laser, each bead emits a unique code image that is detected

46 by Illumina’s BeadXpress Reader System.

$47 \quad$ DNA extraction with VeraCode microbeads

48 Tubes containing the VeraCode microbeads were centrifuged in a microcentrifuge at greater than 49 or equal to $10,000 \mathrm{rpm}$. Most of the $70 \%$ ethanol was removed from the tubes, leaving $\sim 150 \mu \mathrm{L}$ 50 of residual $70 \%$ ethanol with the 40,000 beads. Subsequently, $200 \mu \mathrm{L}$ of peripheral blood was 51 added to the $70 \%$ ethanol and bead mixture using a $1 \mathrm{~mL}$ pipet and mixed thoroughly. The 52 peripheral blood specimens containing the bead mixtures were then extracted on the Qiagen 53 BioRobot EZ1 Workstation using the EZ1 DNA Blood $350 \mu \mathrm{L}$ Kit (Valencia, CA) following the 54 manufacturer's standard protocol with a $200 \mu \mathrm{L}$ elution volume. The eluate containing the 55 VeraCode beads was transferred to the well of a 96-well round bottom microplate (Corning Inc., 56 Corning, NY). A KingFisher 96 pin magnet head with a tip comb (Thermo Fisher Scientific, 57 Waltham, MA) was used to remove residual Qiagen magnetic beads that could potentially 58 interfere with the BeadXpress Reader System.

59 Determination of the number of VeraCode microbeads carried through the DNA $60 \quad$ extraction process 
61 On seven independent days, VeraCode microbeads were added to three peripheral blood

62 specimens. As is outlined in Table 1, the same sets of peripheral blood specimens were used for

63 two or three days. Following the extraction process described in the above section, the beads

64 were transferred to a 76.2 x $25.4 \mathrm{~mm}$ standard glass microscope slide and a $24 \times 50 \mathrm{~mm}$ cover

65 glass was used. The VeraCode microbeads were counted using a microscope under 100X

66 magnification.

\section{$\underline{\text { Utilization of VeraCode microbeads for specimen identification }}$}

68 Six peripheral blood specimens submitted for cystic fibrosis gene mutation testing underwent

69 DNA extraction as described above with and without VeraCode microbeads added to the

70 peripheral blood; beads containing a unique bead identification code were added to each

71 peripheral blood specimen. DNA eluates with and without the beads were tested with the Cystic

72 Fibrosis Genotyping Assay (Abbott Laboratories, Abbott Park, IL). In brief, this assay detects 32

73 cystic fibrosis transmembrane conductance regulator (CFTR) gene mutations via multiplex PCR

74 amplification and oligonucleotide ligation assays followed by detection via capillary

75 electrophoresis on an Applied Biosystems 3130xl Genetic Analyzer (Life Technologies

76 Corporation, Carlsbad, CA). The resulting electropherograms were interpreted and evaluated for

77 equivalency by two molecular pathologists (JDM and IS). A plate containing the residual DNA

78 specimens with and without the beads was covered, frozen, and shipped to Illumina, Inc. to use

79 the BeadXpress Reader System in order to determine the bead number associated with each

80 specimen. We required that $\geq 20$ beads with the same barcode be detected by the instrument for

81 definitive specimen identification for all experiments. In internal experiments, Illumina has

82 determined that the bead mis-identification rate is under $0.5 \%$, suggesting that a requirement for

8320 beads for sample identification is more than sufficient. 
85 known cancer diagnosis, and for three of the specimens, we spiked in a cell line containing the $86 I G H-B C L 2$ translocation at a 1:10,000 dilution to generate positive sensitivity controls near the

87 lower limit of detection of the $I G H-B C L 2$ translocation assay. DNA extraction was performed as

88 described above with and without VeraCode microbeads added to each of the six peripheral

89 blood specimens. Beads containing a unique bead identification code were added to each

90 peripheral blood specimen. DNA eluates with and without the beads were tested with our assay

91 used to detect $I G H-B C L 2$ translocations involving the major breakpoint region, minor cluster

92 region and intermediate cluster region of $B C L 2$. This assay uses multiplex, nested PCR followed

93 by detection of amplification products via agarose gel electrophoresis. The resulting gel

94 photographs were interpreted and evaluated for equivalency by two molecular pathologists (JDM

95 and IS). A plate containing the residual DNA specimens with and without the beads was

96 covered, frozen, shipped to Illumina, Inc., and scanned on the BeadXpress Reader System in

97 order to determine the unique bead identification code associated with each specimen. The

98 group using the BeadXpress Reader System was blinded to which specimens had beads added

99 and to which bead identification code was used in each sample.

$100 \quad$ Examination of VeraCode bead and DNA stability

101 Three peripheral blood specimens were used for this portion of the study - each was submitted to

102 our molecular pathology laboratory for testing with either the Cystic Fibrosis Genotyping Assay

103 described above, the quantitative JAK2 V617F MutaQuant assay (Ipsogen, Stamford, CT), or our

104 laboratory-developed Fragile X syndrome assay. The JAK2 V617F MutaQuant assay is an

105 allele-specific, real-time PCR assay that quantifies both JAK2 V617F and corresponding

106 wild-type alleles. Our laboratory-developed Fragile X syndrome assay uses PCR optimized for 
107 GC-rich amplicons followed by capillary electrophoresis on an Applied Biosystems 3130xl

108 Genetic Analyzer to detect $(\mathrm{CGG})_{\mathrm{n}}$ trinucleotide repeat expansions in the 5' untranslated region

109 of FMR1. Each specimen was assigned a unique bead identification code. Beads with this

110 identification code were added to four aliquots of each peripheral blood specimen, and the

111 specimens were extracted as described above. Eluates containing the beads were incubated at

$11225^{\circ} \mathrm{C}$. Two aliquots of each specimen were removed after 50 days and 90 days. Each specimen

113 was re-tested using the same assay for which the specimen was originally submitted, and the

114 results were interpreted and evaluated for equivalency by two molecular pathologists (JDM and

115 IS). A plate containing the DNA specimens with beads was covered, frozen, and shipped to

116 Illumina, Inc. to use the BeadXpress Reader System in order to determine the bead identification

117 code associated with each specimen. The group using the BeadXpress Reader System was

118 blinded to which bead identification code was added to each specimen.

119 Accelerated stability calculations used the $\mathrm{Q}_{10}$ model, a simplification of the Arrhenius

120 equation approach (

121 well-characterized reagent at $\leq 4^{\circ} \mathrm{C}$ based on stability at the elevated temperature of $25^{\circ} \mathrm{C}$. The

$122 \mathrm{Q}_{10}$ value equals 2. Under the $\mathrm{Q}_{10}$ model, the time relationship between the accelerated

123 temperature (Taccel) and the storage temperature (Tstorage) is given by the equation Q Factor $=$

$1242^{(\text {(Taccel-Tstorage) } / 10)}$; therefore, for this experiment the Q Factor $=2^{((25-4) / 10)}=4.3$. One day at $25^{\circ} \mathrm{C}$ is

125 equivalent to $\sim 4$ days at $4^{\circ} \mathrm{C}$.

126

127 Results

128 We initially observed that VeraCode microbeads containing a holographic identification code

129 could be added to whole blood specimens and subsequently carried through the genomic DNA 
130 extraction process on instruments that use magnetic glass particles. We observed equivalent

131 results using the BioRobot EZ1 Workstation (Qiagen) and MagNA Pure LC (Roche Diagnostics

132 Corporation, Indianapolis, IN), and used the BioRobot EZ1 Workstation for subsequent work

133 described in this manuscript. Given potential applications for specimen identification and

134 quality assurance in the molecular pathology laboratory, we performed a set of experiments

135 designed to evaluate the potential feasibility of using the VeraCode microbeads to track

136 specimens in a clinical molecular laboratory. We found that a sufficient number of microbeads

137 are consistently carried over during genomic DNA extraction to allow specimen identification,

138 that the beads do not interfere with several different molecular assays, and that the beads and

139 genomic DNA are stable when stored together over extended periods of time.

140 We first evaluated if a sufficient number of microbeads would be carried through the

141 DNA extraction procedure to be read by the BeadXpress Reader System (Illumina), thereby

142 allowing identification of the specimen being tested. Although in practice even a single bead can

143 be read and detected by the system, we conservatively established that at least 20 microbeads

144 with the same identification code should be present to allow reliable detection with this system.

145 As is shown in Table 1, well in excess of 20 microbeads were carried through the extraction

146 procedure using multiple different specimens over multiple days. In total, 21 individual

147 extractions were evaluated, yielding a median of 242 microbeads per extraction (range 83 -

148 1096). Given that 40,000 microbeads are added to the initial whole blood aliquot, the efficiency

149 of carryover to the DNA eluate is low, but a sufficient number of beads are consistently

150 transferred to allow detection by the system. Of note, the measured DNA yield of extractions

151 that included the beads was generally lower than that of the same specimen extracted without

152 microbeads - average of $57 \mathrm{ng} / \mu \mathrm{L}$ without beads and $34 \mathrm{ng} / \mu \mathrm{L}$ with beads (Table 2 ). PCR can 
153 be reproducibly performed on nanogram quantities of genomic DNA, and our laboratory

154 generally uses between 1-1,000 ng of genomic DNA per PCR reaction. Therefore, the DNA

155 concentration and total yield were for sufficient for molecular testing, and we usually had

156 micrograms of residual DNA. It is unclear why the presence of the microbeads during the

157 extraction process reduced the amount of DNA in the eluate. It is possible that some of the DNA

158 was bound to the glass microbeads and therefore not available to be measured in solution.

159 Collectively, these data indicate that a sufficient number of microbeads are carried through the

160 DNA extraction procedure to be read by the BeadXpress Reader System, and that inclusion of

161 the microbeads does not appreciably impact the DNA extraction process.

162 We subsequently examined whether inclusion of the VeraCode microbeads affected the

163 performance of two clinical assays commonly performed in our molecular pathology laboratory

164 and whether the beads could be used to track DNA specimens within our laboratory. We

165 hypothesized that the beads would not interfere with molecular assays because they are

166 commonly used in related genotyping assays (Lin et al. 2009). Six peripheral blood specimens

167 submitted for CFTR mutation testing underwent DNA extraction with and without VeraCode

168 microbeads added to the peripheral blood. A unique bead identification code was added to each

169 peripheral blood specimen, essentially adding a readily readable molecular barcode to the

170 specimen. DNA eluates with and without the beads were tested with our laboratory's cystic

171 fibrosis carrier screening assay. The resulting data were interpreted by two molecular

172 pathologists, and the results of the cystic fibrosis assays were the same in the paired peripheral

173 blood specimens with and without the beads (Table 3). In addition, peak positions and heights

174 were equivalent in the specimens with and without beads (Figure 1 illustrates a representative

175 example), indicating that the presence of the microbeads does not significantly interfere with the 
176 performance of this assay. Subsequently, members of our group blinded to which bead was

177 added to each specimen successfully used the BeadXpress Reader System to identify the correct

178 bead identification code originally added to each specimen (Table 3).

179 We also performed a similar set of experiments to that described above with the cystic

180 fibrosis carrier assay, using our laboratory assay for the qualitative detection of $I G H-B C L 2$

181 translocations by PCR. As is seen in Table 3 and Figure 2, the results were the same for all six

182 specimens tested and assay performance was equivalent. Likewise, we were able to re-identify

183 in a blinded manner which bead was added to which specimen.

184 The cystic fibrosis and $I G H-B C L 2$ experiments also allowed examination of time

185 required for operation of the BeadXpress Reader System. Since the plates containing the

186 specimens with microbeads can be directly loaded into a drawer in the BeadXpress Reader

187 System after thawing, minimal hands-on time is required for this step. Once the instrument is

188 initialized, the time to get results for 1,12 , or 96 specimens is approximately 5,10 , and 60

189 minutes, respectively.

190 Finally, to examine the long-term stability of the VeraCode microbeads we used

191 accelerated stability experiments, and we also evaluated the integrity of the DNA under these

192 conditions. We selected three peripheral blood specimens submitted to our molecular pathology

193 laboratory for testing with either the Cystic Fibrosis Genotyping Assay, a quantitative JAK2

194 V617F MutaQuant assay, or our laboratory-developed Fragile X syndrome assay. Beads with a

195 number specific to each specimen were added to multiple aliquots of each peripheral blood

196 specimen, and DNA was extracted from the specimens. Eluates containing the beads were

197 incubated at $25^{\circ} \mathrm{C}$, which was our elevated storage temperature. Using the $\mathrm{Q}_{10}$ model, one day at

$19825^{\circ} \mathrm{C}$ is equivalent to just over four days at or below $4^{\circ} \mathrm{C}$. Two aliquots of each specimen were 
199 removed from incubation at $25^{\circ} \mathrm{C}$ after 50 days and 90 days, which is projected to represent $4^{\circ} \mathrm{C}$

200 incubation for 215 days and 387 days. Each specimen was re-tested using the same assay for

201 which the specimen was originally submitted, and the results were equivalent in both replicates

202 at both time points. In addition, the correct bead number associated with each replicate was

203 identified in a blinded manner. We note that the accelerated stability calculations using the $\mathrm{Q}_{10}$

204 model is a conservative approach in the assignment of $\mathrm{Q}_{10}=2$. In addition, if this method is used

205 to calculate the stability reagents stored at $-15^{\circ} \mathrm{C}$ or below, additional stability may be conferred

206 due to the phase transition. Collectively, these data indicate that the beads and the DNA in the

207 eluate are stable for at least one year at $4^{\circ} \mathrm{C}$ and possibly longer when stored frozen.

208

209 Discussion

210 In this report, we evaluated the feasibility of using the VeraCode microbeads to track DNA

211 specimens in the clinical molecular laboratory. We found that a sufficient number of microbeads

212 are consistently carried over during genomic DNA extraction to allow specimen identification,

213 that the beads do not appear to interfere with several different molecular assays, and that the

214 beads and genomic DNA are stable when stored together over extended periods of time. The

215 beads function as an internal, easily readable specimen barcode. This method may provide an

216 additional approach to identifying DNA specimens and minimizing errors associated with

217 molecular laboratory testing. Presently, this approach could be used to recheck the identity of

218 DNA specimens that give unexpected results or to check specimens at a set interval as part of the

219 laboratory quality assurance program. This would be a novel way to monitor for possible

220 specimen mix-ups. Although the hands-on time and scanning time are reasonable, our 
221 experience suggests that it would not be practical to examine every specimen tested by a busy

222 molecular pathology laboratory with our method.

223 We suggest that this approach represents a novel mechanism to track DNA specimens in

224 the molecular pathology laboratory, and the data presented provide a proof of concept that such

225 an approach is possible. However, a couple of technical issues related to the microbead size

226 currently limit the potential utility of this approach. The present microbeads are too large to

227 co-elute with DNA using column-based extraction methods, and consequently this microbead

228 tracking system cannot be used with many DNA and RNA extraction methods. Likewise, the

229 relatively large size of the microbeads makes it difficult to consistently transfer a sufficient

230 number of beads from the DNA eluate to downstream assay steps due to the microliter volumes

231 typically used during molecular testing. As an example, we found that addition of DNA from the

232 tube containing the DNA eluate with microbeads to a standard PCR reaction did not reliably

233 result in the transfer of a sufficient number of beads to be detected by the barcode reader.

234 However, further manipulation of the beads or extraction process may allow for these limitations

235 to be overcome and for this application to become more broadly applied.

236 Other factors must be addressed and studies performed before the method can be

237 incorporated into routine clinical practice. First, the BeadXpress Reader System and associated

238 VeraCode microbeads were not designed for this application. Consequently, the final retail list

239 price of the reader, $\$ 98,000$, was likely higher than an instrument designed solely for

240 applications described in this manuscript. Furthermore, the configuration of VeraCode

241 microbeads used in this study was custom prepared and is not commercially available at this

242 time. Second, we evaluated this method to track DNA specimens extracted from peripheral

243 blood. For this method to be applied to all areas of the molecular pathology laboratory, further 
244 examination of RNA extracted specimens would need to be performed. Likewise, specimens

245 other than peripheral blood would need to be evaluated, and in the case of tissue specimens, the

246 protocol would likely need to be modified to avoid, among other issues, destruction of the glass

247 microbeads during the homogenization process. Third, we examined four assays in this study,

248 and a thorough validation would require examination of a larger number of qualitative and

249 quantitative assays with associated statistical assessment of interference.

250 In summary, we have demonstrated a proof of concept that glass microbeads with a

251 holographic numeric code can be used to barcode DNA eluates from peripheral blood specimens.

252 This represents a novel way to track DNA specimens in the molecular pathology laboratory, and

253 we suggest that with appropriate modifications and further evaluation such an approach could

254 minimize the potential for errors associated with molecular laboratory testing. 


\section{References}

258 Hemmerich KJ. 1998. General Aging Theory and Simplified Protocol for Accelerated Aging of 259 Medical Devices. Medical Plastics and Biomaterials 5:16-23.

260 Hofgartner WT, and Tait JF. 1999. Frequency of problems during clinical molecular-genetic 261 testing. Am J Clin Pathol 112:14-21.

262 Lin CH, Yeakley JM, McDaniel TK, and Shen R. 2009. Medium- to high-throughput SNP 263 genotyping using VeraCode microbeads. Methods Mol Biol 496:129-142.

264

265

266 


\section{Table $\mathbf{1}_{\text {(on next page) }}$}

Number of Veracode microbeads carried through the DNA extraction process. 


\begin{tabular}{|l|l|l|l|l|l|l|l|l|}
\hline \multicolumn{3}{|c|}{ Days 1, 2 and 3 } & \multicolumn{3}{c|}{ Days 4 and 5 } & \multicolumn{3}{c|}{ Days 6 and 7 } \\
\hline Specimen 1 & Specimen 2 & Specimen 3 & Specimen 4 & Specimen 5 & Specimen 6 & Specimen 7 & Specimen 8 & Specimen 9 \\
\hline 534 & 464 & 743 & 108 & 203 & 135 & 231 & 295 & 408 \\
\hline 1096 & 778 & 362 & 168 & 242 & 83 & 140 & 174 & 101 \\
\hline 105 & 279 & 257 & \multicolumn{3}{|l|}{} \\
\hline
\end{tabular}




\section{Table 2 (on next page)}

Effect of including Veracode microbeads during DNA extraction on resulting DNA concentration. 


\begin{tabular}{|l|l|l|}
\hline Specimen & DNA concentration when & DNA concentration when \\
& extracted without beads & extracted with beads \\
\hline 1 & $43 \mathrm{ng} / \mu \mathrm{L}$ & $22 \mathrm{ng} / \mu \mathrm{L}$ \\
\hline 2 & $51 \mathrm{ng} / \mu \mathrm{L}$ & $33 \mathrm{ng} / \mu \mathrm{L}$ \\
\hline 3 & $44 \mathrm{ng} / \mu \mathrm{L}$ & $24 \mathrm{ng} / \mu \mathrm{L}$ \\
\hline 4 & $73 \mathrm{ng} / \mu \mathrm{L}$ & $44 \mathrm{ng} / \mu \mathrm{L}$ \\
\hline 5 & $49 \mathrm{ng} / \mu \mathrm{L}$ & $25 \mathrm{ng} / \mu \mathrm{L}$ \\
\hline 6 & $79 \mathrm{ng} / \mu \mathrm{L}$ & $53 \mathrm{ng} / \mu \mathrm{L}$ \\
\hline
\end{tabular}




\section{Table 3 (on next page)}

Using Veracode microbeads to identify DNA specimens and examining their effect on two molecular pathology assays. 


\begin{tabular}{|l|l|l|l|l|l|l|}
\hline Specimen & Bead number & Bead number & Assay & Assay result with & Assay result without & Equivalent \\
number & added & detected & & microbeads added & beads added & amplification \\
\hline 10 & 4096 & 4096 & CF32 & homozygous delF508 & homozygous delF508 & Yes \\
\hline 11 & 272 & 272 & CF32 & homozygous delF508 & homozygous delF508 & Yes \\
\hline 12 & 40 & 40 & CF32 & homozygous delF508 & homozygous delF508 & Yes \\
\hline 13 & 136 & 136 & CF32 & no mutations detected & no mutations detected & Yes \\
\hline 14 & 2560 & 2560 & CF32 & no mutations detected & no mutations detected & Yes \\
\hline 15 & 1040 & 1040 & CF32 & no mutations detected & no mutations detected & Yes \\
\hline 16 & 1040 & 1040 & BCL2 & $I G H-B C L 2$ detected & $I G H-B C L 2$ detected & Yes \\
\hline 17 & 2560 & 2560 & BCL2 & $I G H-B C L 2$ not detected & $I G H-B C L 2$ not detected & Yes \\
\hline 18 & 136 & 136 & BCL2 & $I G H-B C L 2$ detected & $I G H-B C L 2$ detected & Yes \\
\hline 19 & 40 & 40 & BCL2 & $I G H-B C L 2$ not detected & $I G H-B C L 2$ not detected & Yes \\
\hline 20 & 272 & 272 & BCL2 & $I G H-B C L 2$ detected & $I G H-B C L 2$ detected & Yes \\
\hline 21 & 4096 & 4096 & BCL2 & $I G H-B C L 2$ not detected & $I G H-B C L 2$ not detected & Yes \\
\hline
\end{tabular}

\section{CF32 - Abbott Laboratories Cystic Fibrosis Genotyping Assay}

BCL2 - Laboratory-developed assay for detection of IGH-BCL2 translocations 


\section{Figure 1}

Representative capillary electropherograms from a multiplex PCR amplification and oligonucleotide ligation assay to detect 32 different mutations in the CFTR gene.

The presence of beads during the extraction process and downstream steps (panel A) does not appear to affect either peak height or assay results when compared to analysis of the same specimen without beads (panel B). No CFTR mutations were detected in the specimen with or without the beads and each of the peaks is present at the same position.

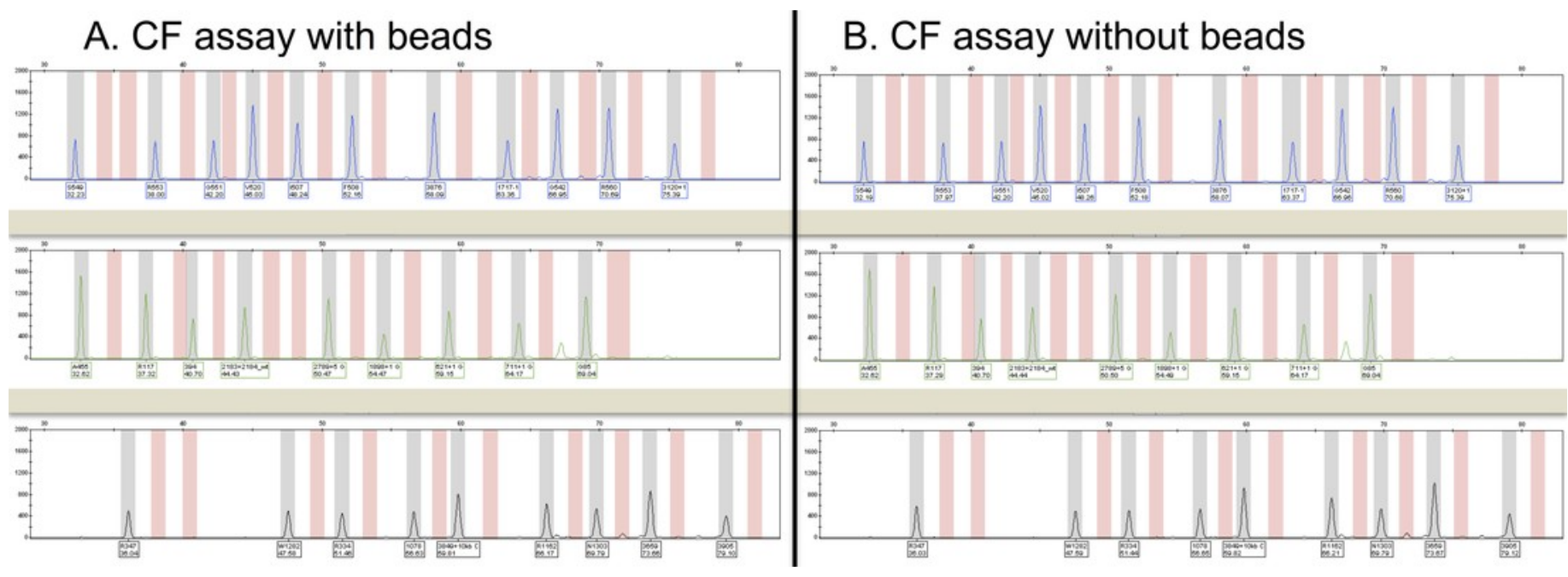




\section{Figure 2}

Agarose gel electrophoresis demonstrating control amplification reactions for a PCR-based IGH-BCL2 translocation assay.

The presence of beads during the extraction process and downstream steps does not significantly affect the control amplification for this assay. This control amplicon is a 270 bp product derived from the F5 gene.

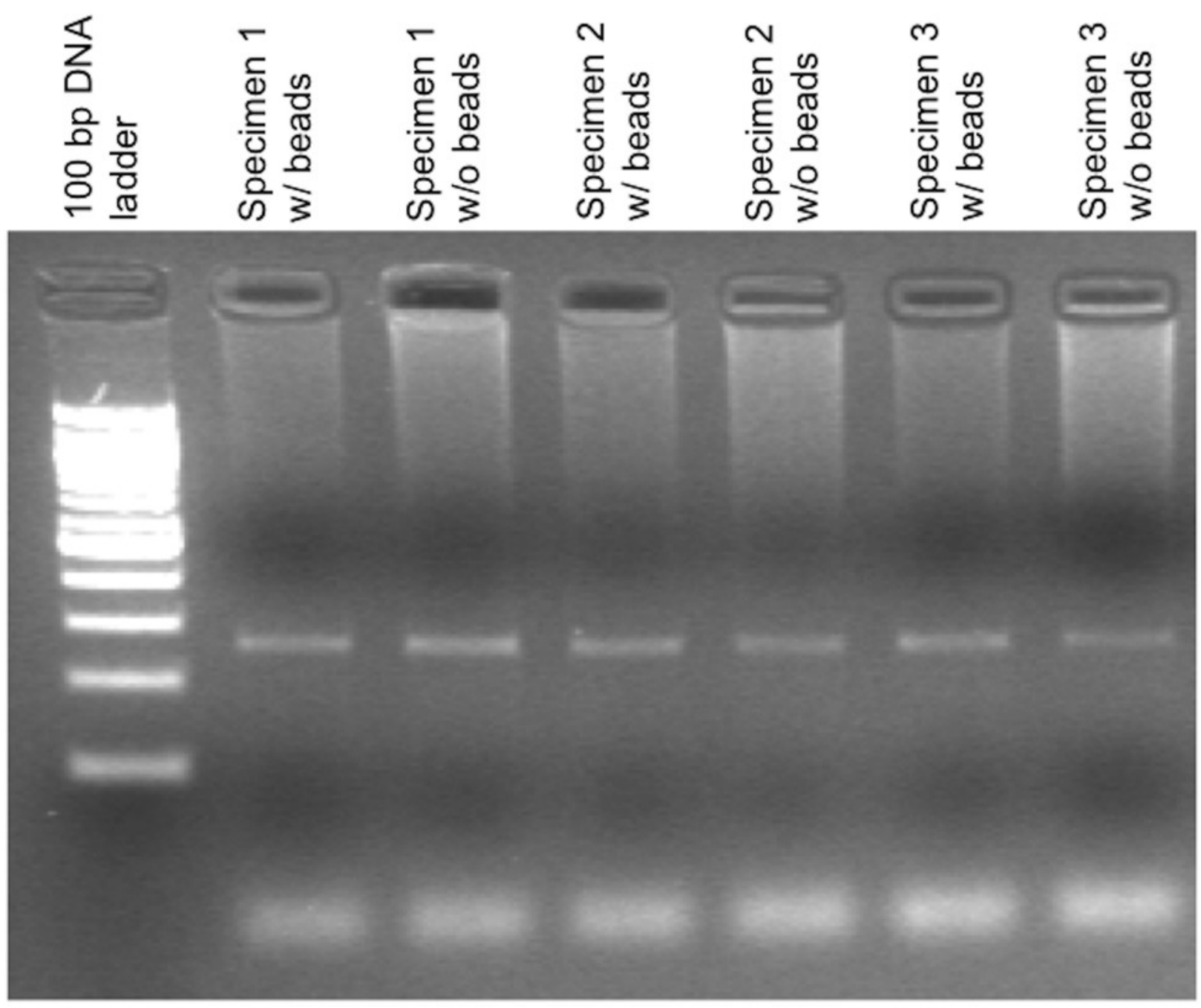

